Meta

Journal des tradlucteurs

Translators' Journal

\title{
De la translittération en psychanalyse
}

\section{Jean Allouch}

Volume 27, numéro 1, mars 1982

Psychanalyse et traduction

URI : https://id.erudit.org/iderudit/001874ar

DOI : https://doi.org/10.7202/001874ar

Aller au sommaire du numéro

\section{Éditeur(s)}

Les Presses de l'Université de Montréal

\section{ISSN}

0026-0452 (imprimé)

1492-1421 (numérique)

Découvrir la revue

\section{Citer cet article}

Allouch, J. (1982). De la translittération en psychanalyse. Meta, 27(1), 77-86.

https://doi.org/10.7202/001874ar d'utilisation que vous pouvez consulter en ligne.

https://apropos.erudit.org/fr/usagers/politique-dutilisation/ 


\section{DE LA TRANSLITTÉRATION EN PSYCHANALYSE*}

JEAN ALLOUCH

Les termes vraiment termes sont autres que les termes constants.

TAO TO KING** ...voir dans le marc de café n'est pas lire dans les hiéroglyphes...

J. LACAN

Écrits, p. 510

«Ose ordonner la réforme de notre orthographe et le mensonge abécédaire qui prépare à tous les mensonges ne déformera plus les jeunes esprits» : cette invite que le grammairien Urbain Domergue adressait en 1805 à l'empereur ${ }^{1}$, comment ne pas noter qu'elle se portait au lieu exact d'où elle pouvait trouver un commencement de réalisation? La distribution des diverses écritures s'avère en effet non pas isomorphe à l'éparpillement des langues mais coextensive au développement des empires et des religions. Ce fait est suffisant à désigner la distinction essentielle de ce qui relève de l'écrit et de ce qui est de l'ordre des langues en tant que parlées. Antérieure à l'établissement d'écritures de langues, l'existence de systèmes de notation d'éléments articulés, en l'occasion même systématiquement ordonnés tels les hexagrammes de la méthode divinatoire chinoise du Yi King, confirme cette distinction, invite à en maintenir le tranchant.

Ainsi une définition de l'écriture du type de celle que propose Marcel Cohen à savoir comme «instrument qui double le langage en présentant à la vue ce que celui-ci fournit à l'oreille " apparaît soumise à un préjugé ethnocentrique, visant non pas à cerner ce qu'est l'écriture mais énonçant bien plutôt ce qu'à son sens elle se devrait d'être. Cette morale - Marcel Cohen va jusqu'à écrire le terme de "loyauté ${ }^{2}$ - est celle-là même qui souffle à Urbain Domergue l'expression de «mensonge abécédaire», expression étonnante pour le moins; car contrairement à ce qu'à l'entendre vous pourriez en conclure elle ne dénonce pas l'abécédaire comme tel mais son usage jugé par le grammairien incorrect. L'abus tiendrait au non-respect du «principe de l'écriture alphabétique ${ }^{3}$, lequel se formule ainsi : représenter phonographiquement le langage. Pourtant même à ne considérer ce principe que comme l'aboutissement d'un prétendu progrès, il est patent que les diverses réalisations de l'écriture alphabétique lui font ellesmêmes objection en ce qu'elles ne satisfont que très approximativement l'impé-

\footnotetext{
* Ce texte a été présenté dans le cadre du IX congrès de l'École freudienne de Paris sur "La transmission de la psychanalyse", il a été publié dans les Actes du Congrès par le bulletin interne de l'École: Les Lettres de l'École freudienne ${ }^{\circ} 25(1), 1978$.

* Traduction Duyvendak, Librairie d'Amérique et d'Orient, 1975.

1. Cité par Marcel Cohen (1958): «La grande invention de l'écriture ", Klincksieck, 1958.

2. Ibid. p. 317 .

3. Ibid. p. 222
} 
ratif phonographique. En témoigne la distinction à laquelle sont contraints les spécialistes d'une orthographe dite phonologisante qui note par une même lettre un phonème ayant une prononciation différenciée et d'une orthographe phonétisante où les variations de prononciation sont notées par des changements de lettres ${ }^{4}$.

Il n'est pas sûr que l'alphabet latin fournisse le système de notations le plus approprié à une écriture phonétique dont la règle - un signe par son, une valeur unique pour chaque signe - était déjà posée par la grammaire de Port-Royal. Otto Jespersen ne s'est pas contenté de faire une critique serrée des inconvénients de l'alphabet latin, il a su également proposer un autre ensemble de notations beaucoup plus rigoureux. Mais le gain ainsi obtenu dans l'application de la règle phonétique, gain considérable puisqu'il ne s'agit de rien de moins que d'une écriture susceptible de noter n'importe quelle langue, objet idéal pour les communications internationales et la réalisation des machines qui s'y emploient, se paye d'une complexité telle des notations que leur usage perd toute chance de pouvoir jamais être adoptés. Si l'alphabet de l'Association Phonétique Internationale s'est avéré plus utilisable, c'est bien d'avoir accepté de mettre de l'eau dans le vin de l'exigence phonétique - graphies complexes, accents et non sans qu'il soit apparu que cet alphabet loin de satisfaire à tous les besoins se révèle d'autre part, aux dires des spécialistes eux-mêmes, d'un emploi fort malaisé.

Cette difficulté interne à l'écriture dite phonétique se redouble d'un autre point d'achoppement. En effet la prononciation n'est pas réductible à un phénomène stable et l'écriture, à la suivre de trop près, édifierait dès lors des textes qui deviendraient - tels pour nos contemporains ceux de l'ancien français sinon illisibles du moins déchiffrables au prix d'instructions supplémentaires, c'est-à-dire de ce que l'écriture phonétique vise précisément à supprimer. Ainsi une écriture qui prend appui de la phonétique pour établir les règles de ses tracés peut faire miroiter à son horizon l'idée qu'à la suivre dans sa manière, elle rendra simple l'acte de lecture, même s'il s'agit pour un individu donné d'une langue inconnue. Il reste que cette écriture de toutes les langues est à proprement parler impossible, terme particulièrement approprié ici en sa définition lacanienne, cette écriture en effet ne cesse pas de ne pas s'écrire. Et de fait, notre alphabet occidental à peine constitué nous voyons aussitôt apparaître des procédés sténographiques, c'est-à-dire un type de notations qu'on qualifie à tort d'idéographique puisqu'il s'agit plus justement de ce que les Allemands désignent du terme de Wortschrift, d'une écriture de mots. Il n'est que d'ouvrir les notes de Freud sur le cas de l'Homme aux rats pour mesurer l'importance de ce mode d'écriture dont chacun fait un usage quotidien. L'impossibilité d'une

4. Exemple d'une orthographe phonologique donné par M. Cohen: en français la lettre $x$ note la consonne complexe $k s$ dans toxique ou axiome et note également $g z$ dans exact ou hexagone, en allemand ce qui s'écrit $c h$ se prononce différemment dans buch ou hoch et dans Dich ou echt. Exemple d'écriture phonétisante: on écrit en latin implere au lieu de inplere, accidere au lieu de adcidere.

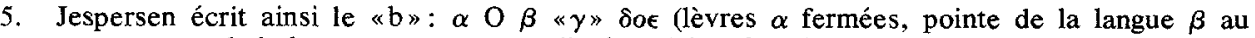
repos, corps de la langue $\gamma$ au repos, voile du palais $\delta$ fermé, glotte $\epsilon$ en vibration). 
écriture phonétique dite universelle apparaitra moins étrange à souligner ce qui se présente comme la visée de l'opération à savoir la suppression de l'équivoque du champ de l'écrit; mais tout aussi important est le procédé dont on attend pareille suppression, puisqu'il s'agit de référer l'écrit à un objet extérieur à son domaine, soit le phonétique dont on espère qu'il fera loi en ses tracés.

$\mathrm{Au}$ cours du séminaire du 20 mars 1957, juste après qu'ait été publié dans la revue La psychanalyse $\left(\mathrm{n}^{\circ} 2\right)$ le texte de Lacan sur «La lettre volée », un participant a soulevé cette question centrale de l'orthographie. Il n'est pas possible de reprendre ici dans son détail l'ensemble de la discussion ${ }^{6}$. Son intérêt tient à ce que l'enseignement de Lacan fait de «La lettre volée» quelque chose qui a valeur non pas expérimentale mais véritablement d'expérience. Celle-ci consiste à réduire le réel de ce qui est noté à un pur hasard; il s'avère alors que cette réduction, loin d'abolir toute orthographie, démontre l'ancrage de la loi orthographique dans la graphie elle-même. Or s'il n'est d'orthographie que de la graphie, c'est en tant que chacun de ses éléments, comme non identique à soi, est porteur, quoi qu'il en soit, d'équivocité. La simplicité du répartitoire des $\alpha \beta \gamma \delta$ est solidaire de la non-discrimination des différentes sortes d' $\alpha$, de $\beta$, de $\gamma$ et de $\delta$.

On nommera transcription un mode de notation qui tente de fonder son orthographe à partir d'un objet extérieur au champ proprement graphique; l'idéal de la transcription est d'amener l'écriture à être, comme l'écrit Fouché la mise en ouvre "d'un code de signes ayant une valeur absolue et indépendante de la graphie ${ }^{7}$. Soit à reprendre ici l'exemple de J.G. Février dans sa monumentale Histoire de l'écriture ${ }^{8}$, le mot grec $\sigma v \nu \tau \rho \mathrm{i} \beta \omega$ : sa transcription en caractères latins s'écrira SINDRIVO, ce qui correspond à ce qu'on croit savoir de sa prononciation effective. Il est envisageable cependant non plus de transcrire mais de translittérer ce même mot: on écrira alors SYNTRIBO. Cette graphie respecte la correspondance lettre à lettre des alphabets grec et latin, rend ainsi l'étymologie ancienne lisible en la nouvelle inscription, mais implique par contre, s'il s'agit de la lire à haute voix, que le lecteur dispose d'instructions supplémentaires qui lui indiqueront qu'à vouloir s'en tenir à la prononciation ancienne, il devra prononcer $t: d$ et $b: v$. La translittération est définie par $M$. Cohen ${ }^{9}$ comme «un transfert caractère à caractère d'une écriture dans une autre sans aucune interprétation». Le terme d'interprétation est ici à entendre comme la conjecture qu'est amené à avancer le transcripteur lorsqu'il a affaire à une écriture dont la prononciation est de lui mal connue; le "sans interprétation» veut donc dire ici sans égards pour la prononciation. La translittération en donnant à chaque lettre ou signe syllabique un correspondant dans un autre alphabet construit un écrit à partir de l'écrit, procède pour ce faire au coup par coup, c'est-à-dire compte un à chacune de ses opérations.

Ainsi défini, ce terme de translittération désigne de façon adéquate le rapport qu'entretiennent deux à deux en leur succession les différentes écritures de

6. «Le monter en épingle du transfert». Lettres de l'École freudienne, $\mathrm{n}^{\circ} 21$.

7. P. Fouché : Traité de prononciation française, p. VI. Cité par le Robert, article: Transcrire.

8. J. G. Février : Histoire de l'écriture, p. 550.

9. M. Cohen : La grande invention de l'écriture, p. 309. 
la série au hasard développée dans le séminaire sur la lettre volée. Or, si chacun de ces alphabets inscrit correctement cette série au hasard, on sait que ce n'est cependant qu'avec le second d'entre eux - soit la définition des (1) (2) (3) qu'apparaît une loi des successions c'est-à-dire une orthographie. Ceci démontre étant donné que la première série d'inscriptions - celle des + - est de l'ordre d'une transcription, qu'il n'y a d'orthographie repérable qu'avec l'intervention, qui ne peut être que seconde, de la translittération.

L'opposition de la transcription et de la translittération appelle la remarque suivante : si la transcription ramène l'écrit du côté d'une écriture phonétique, la translittération par contre l'en éloigne. Mais l'en éloignant, vers quoi tend-elle? Il y a lieu ici de précisément répondre : vers l'idéographie.

L'écriture idéographique est distincte de l'écriture phonétique en ce que, comme l'écriture synthétique, elle est une écriture du sens et non pas du son. Cependant alors que l'opération de l'écriture synthétique est fort joliment épinglée par J. Février du terme de «suggestion », l'intérêt de l'idéographie tient à ce que limitant cet appui pris sur le sens au sens des mots, elle réalise un mode de l'écriture à la fois indépendant de la prononciation et proche du parler. Elle offre ainsi, de cet appui pris sur le sens, un mode de l'écriture virtuellement généralisable. On cite l'exemple d'une histoire japonaise écrite en 712 avec les caractères chinois.

Il est de fait toutefois que l'écriture chinoise tout en ayant servi à noter un grand nombre de langues ne s'est jamais imposée, quoiqu'on l'ait ainsi qualifiée, comme un véritable «esperanto pour les yeux». Fondamentalement la raison de ceci n'est pas à chercher du côté des problèmes - certes non négligeables soulevés par l'emprunt des caractères chinois et leur mise en rapport avec de nouvelles langues, puisque l'emprunt est au contraire la règle la plus générale des rapports langue - écriture. Si la réalisation la plus pure d'une écriture de mots a rencontré une limite à sa propagation, c'est que le phonétisme n'en est pas radicalement absent puisqu'au contraire il participe à l'élaboration ellemême des idéogrammes. La langue chinoise, composée de mots monosyllabiques et invariants s'avère par là très favorable à une écriture de mots; mais ce monosyllabisme en multipliant les homophonies a pour conséquence opposée d'offrir à l'écriture des équivoques sonores dont elle a su se servir pour l'établissement de ses caractères. Le procédé alors utilisé est celui dit du «rébus à transfert» par lequel un graphisme déjà codifié comme écriture d'un mot est repris pour noter un mot de sens différent mais homophone. En retour une écriture ainsi constituée fait se porter l'attention sur l'homophonie, donne par là à lire le son et non plus le sens. Le rébus à transfert s'avère donc faire limite interne à l'idéographie. Son intervention, qui est loin d'être négligeable également au sein des autres modes de l'écriture - écriture syllabique, écriture consonantique -, peut être partout définie, J. Février ne manque pas à maintes reprises de le souligner, comme une invitation à l'écriture phonétique.

L'écriture par les choses - rébus - conduit à appréhender les langues comme choses - res - même si elle ne parvient pas à donner de cette façon à l'écriture un fondement qui serait transcription. Si le rébus à transfert se contente d'inviter à l'écriture phonétique, son opération cependant est effective en 
ce qu'il souligne le caractère littéral du signifiant, qu'il distingue du flot des paroles les éléments, terme dont ce n'est pas hasard qu'il se soit forgé à partir de la succession conventionnelle des lettres $l, m, n$, c'est-à-dire comme un effet de l'écrit au niveau de la langue.

La translittération est le nom du rébus à transfert en tant que celui-ci s'avère pouvoir opérer non pas simplement à l'intérieur d'un mode donné de l'écriture mais au travers - durch - de ses divers modes.

$\mathrm{Ce}$ «trans " est d'autant plus envisageable que c'est précisément ce qui est en jeu chaque fois qu'intervient le rébus à transfert. Ainsi peut-on interpréter la fonction des clés dans l'écriture chinoise comme ce qui ferait réponse par une notation supplémentaire du sens à ce qui pourrait se construire d'une écriture autre à partir de l'homophonie. Il n'est d'ailleurs que de pratiquer les jeux de rébus offerts dans les foisonnantes Fliegende Blätter dont chacun dispose, pour toucher du doigt que le rébus, quand aux diverses façons d'écrire, fait feu de tout bois.

Freud, on le sait, non seulement compare mais identifie rêve et rébus. Le rêve est une Bilderschrift, une écriture par images. Ce n'est certes pas dire qu'il ne puisse comporter d'autres éléments que pictographiques : paroles prononcées ou entendues, idéogrammes, signes de ponctuation, distribution signifiante de l'espace, bouts de texte écrits, formules, lettres isolées y cohabitent comme dans le rébus. C'est bien plutôt la prise en compte du rêve au titre d'un rébus qui fera de chacun de ses éléments, pictogramme ou pas, et quel que soit le mode de l'écriture qu'il semble d'abord présentifier, un quelque chose à déchiffrer, c'est-à-dire comme le précise Freud, à être lu non pas d'après sa valeur d'image - Bilderwert - mais d'après sa relation, au demeurant quelconque, à un signe - ce qu'il nomme Zeichenbeziehung. Les éléments du rêve ne livrent leur sens qu'à être repris, il précise ce point, un à un ${ }^{10}$; il n'y a, autrement dit, puisque telle est sa définition, de déchiffrage que de la translittération.

Le travail de l'inconscient qui ne pense, ne calcule ni ne juge, consiste dans le chiffrage. Remarquable est le fait que les traités de cryptographie distinguent deux et seulement deux procédés de chiffrage, procédés qui peuvent bien entendu soit se redoubler chacun, soit se combiner l'un avec l'autre. La transposition garde les lettres du texte dit clair mais en modifie de façon réglée, c'est là la fonction de la clé de la grille ou de la figure, l'ordonnancement. La substitution remplace l'alphabet du texte à cryptographier par un ou plusieurs alphabets de convention. Elle joue donc sur l'axe paradigmatique alors que la transposition réalise un glissement des lettres le long de l'axe syntagmatique. Substitution et transposition, rencontre étonnante, ne sont que les noms de

10. Gesammelte Werke, II/III, p. 283-284. «Le contenu du rêve se donne pour ainsi dire dans une écriture d'images dont les signes sont à transférer un à un dans la langue des pensées du rêve. On serait évidemment induit en erreur si on voulait lire ces signes d'après leur valeur d'image au lieu de les lire d'après leurs relations de signes. " Ces lignes introduisent le terme de rébus ou encore celui de Bilderrätsel - devinette mais aussi énigme par images - termes que Freud pose ici comme équivalents. 
métaphore et métonymie que les cryptologues ont inventés pour désigner ce que Freud a introduit comme lois de l'inconscient dans leur extension la plus générale ${ }^{11}$.

L'expérience de l'inconscient se met ainsi en travers de la question du sens du sens pour lui substituer celle du signe du signe. On se souvient quelle réponse lui a fait Lacan lors de sa première intervention au congrès de Montpellier $^{12}$ : «le signe du signe... c'est que n'importe quel signe fasse aussi bien fonction de tout autre signe précisément de ce qu'il puisse lui être substitué.» Si la fonction du chiffre est ici, comme il le dit encore, «fondamentale», c'est en tant que le chiffre est signe de signe, signe autrement dit translittéré, ou encore signifiant en tant que pris dans «sa fonction de transfert» ${ }^{13}$.

Cette fonction translittérante du signifiant est ce sur quoi il convient que le psychanalyste prenne appui. Un rébus, un cryptogramme, un rêve, un symptôme ça se déchiffre; mieux encore, ça emporte avec soi l'information qui va permettre de trancher quant au caractère correct ou incorrect de ce qui sera ainsi décrypté. Mais n'est-ce pas s'en remettre alors, dira-t-on justement, à ce registre du sens d'abord mis de côté ? Fût-il, ce sens, sexuel, faut-il pour autant nous en tenir là? On sait certes qu'un texte pour se présenter comme sensé peut néanmoins être, tout aussi bien qu'un autre, message chiffré. C'est dire que l'opération du chiffre ne saurait être réduite à celle du sens. Le chiffrage est bien plutôt jouissance et en ce sens obstacle au rapport sexuel.

$Y$ a-t-il une chance - faut-il dire jouichance - avec la supposition d'un sujet au chiffrage, c'est-à-dire avec l'analyse, que le chiffre en vienne à introduire un peu de calcul dans cette «chicane infinie» du savoir inconscient? Chiffrer n'est pas compter; et si «rien de réel n'est communicable en dehors du nombre", est-il envisageable que le chiffre de quoi nous dépendons entièrement puisse "cristalliser" - le mot est de Lacan - la puissance de ce réel?

Cette chimie problématique du chiffre et du nombre est en jeu dans ce que Lacan introduit avec l'écriture des nœuds. Introduit à partir de quoi? De ceci que le signe fait des nœuds. C'est là le symptôme au sens psychanalytique. Et ce que Freud démontre avec ce qu'il nomme Zeichenbeziehung c'est qu'il n'y a nul autre moyen d'avoir accès à ces nœuds que sont les formations de l'inconscient qu'à prendre ces signes à la lettre, un par un, qu'à admettre autrement dit qu'il y a de l'un c'est-à-dire quelque chose sur quoi, différant en cela de la transcription, la translittération ne transige pas.

Si la lettre, support matériel, présentifie la «structure essentiellement localisée du signifiant», la translittération donne le corrélat de cette localisation soulignant qu'en cette structure même le signifiant n'est pas isolable. On sait que c'est là ce qu'a démontré l'analyse de la première scène d'Athalie, tout

11. Cf. Lange et Soudart: Traité de cryptographie, Lib. F. Alcan, 1925. On imagine mal à quel point est difficilement maîtrisable chez l'être parlant cette pratique du chiffre; les bévues s'y développent comme fleurs sous serres, bévues aux conséquences considérables qui vont de la condamnation de Dreyfus à la domination de Rommel en Afrique du nord.

12. Cf. Lettres de l'École freudienne, $\mathrm{n}^{\circ} 15$, p. 69 à 80 et p. 235 à 244.

13. "L'instance de la lettre dans l'inconscient». Écrits, p. 522. 
particulièrement de son célèbre «oui» inaugural, lors du Séminaire sur les psychoses du 6 juin 1956.

La concaténation signifiante donne à lire. Quelqu'un d'aussi averti de l'écriture que $M$. Duras ne manque pas de témoigner que tout un chacun écrit bien au-delà de ce qu'il imagine écrire. Le parler lui-même n'est pas sans charrier de l'écrit. Avec une psychanalyse, ce qui se donne à lire se soutient d'un sujet supposé savoir lire autrement. En ce point le transfert a à recevoir confirmation puisque seule cette confirmation peut faire apparaître qu'il n'est là de sujet que supposé. Pourtant ce lire autrement reste difficile, même à préciser, ce qu'a fait Lacan dans son séminaire de cette année ${ }^{14}$ qu'il s'agit en cette lecture pour le psychanalyste de «manquer autrement». La translittération en tant qu'elle met en jeu non pas un mais deux «alphabets» sollicite le psychanalyste à être dupe de la bonne façon, c'est-à-dire dupe de l'écrit en tant que sa lecture autre ne se supporte que d'un écrit différent.

C'est donc, pour finir, de Lacan en tant que lecteur que nous prendrons enseignement. Dans un exposé présenté à la section clinique j'ai montré que la façon dont Lacan épelle une à une les successives élaborations de celui qu'on s'obstine à nommer «le petit Hans " n'est soumise à rien d'autre qu'à l'écriture de la métaphore telle qu'on la trouve dans "L'instance de la lettre dans l'inconscient». Ce «rien d'autre » à l'instant avancé n'est pas ici effet de style. Il est au contraire décisif en ce qu'il désigne que ce n'est qu'à s'en remettre à cet autre alphabet que la lecture manque autrement, et de ce manque même situe le signifiant phobique comme ce qui supplée au signifiant du nom du père. Il n'est, autrement dit, de démonstration de la nécessité de la phobie que de la mise en rapport - disons ce rapport contingent, pourquoi pas? il ne s'agit pas là du royaume des idées éternelles - de ce qu'écrit l'observation et d'une autre écriture : là où ça ne satisfait pas à cette autre écriture, là même apparaît la phobie comme nécessaire, là-même il convient que ça y satisfasse... quand même. C'est l'appoint de la phobie en tant qu'elle introduit un signifiant à la place du nom du père, qui permet la translittération, formule dont la réciproque s'énonce: c'est de la translittération que le signifiant phobique trouve, avec sa place, sa fonction d'appoint.

Le texte de Lacan sur la jeunesse de Gide nous importe aujourd'hui en tant qu'il présentifie la même exigence, qu'il est non seulement habité mais orienté de part en part, depuis ce point de défaillance de la translittération.

Voici, un bal est donné dans la maison familiale des Gide. Alerté par la rumeur, le jeune André ose furtivement descendre quelques marches de l'escalier; il faut aller voir. Je cite : «Rien n'a l'air coutumier; il me semble que je vais être initié tout à coup à une vie, mystérieuse, différemment réelle, plus brillante et plus pathétique et qui commence seulement lorsque les petits enfants sont couchés. » Une belle dame s'avance vers lui; il ne reconnaît pas, rubans et robe de soie, une amie de sa mère pourtant vue le matin même. Puis, ramené dans son lit, l'esprit tout brouillé, surgit, juste avant qu'il ne sombre dans le sommeil,

14. Le moment de conclure: Sém. du 20.12.1977 et du 10.1.1978. 
cette pensée : «Il y a la réalité et il y a les rêves; et puis il y a la seconde réalité.» L'enfant Gide n'a pas sept ans ${ }^{15}$.

La seconde réalité est ce par rapport à quoi, irréductiblement, dans son rapport aux autres, Gide s'éprouve comme «pas pareil ». De là cet effet de multiplication indéfinie de personnages dont il n'y a aucune raison d'exclure de la liste celui de littérateur. Ainsi par exemple des Faux monnayeurs où un des personnages, Édouard, projette d'écrire un roman dont le titre serait «Les faux monnayeurs » et tient à cette fin un journal où se discutent les enjeux du roman à venir, tandis qu'André Gide, écrivant Les faux monnayeurs propose avec son «roman» un autre livre intitulé Journal des Faux monnayeurs où il écrit, entre mille autres indications semblables, que ce cahier n'est que ce qui «doit devenir le cahier d'Édouard» ${ }^{16}$. Pas plus qu'Édouard, André Walter, Tityre ou Corydon ne s'offrent comme figures d'identification; ainsi Gide note-t-il «Je ne suis pas l'Immoraliste... il me suffit de l'avoir écrit» ${ }^{17}$. Avec ces écrits Gide, sur la scène du monde s'avance démasqué.

Dans son article «Art» de l'Anthropologie structurale $(1958)^{18}$, Claude Levi-Strauss explicite ce que veut dire démasquer. La question se trouve posée à partir d'un ensemble d'analogies qui avaient suscité l'intérêt des anthropologues entre des productions artistiques de populations pourtant fort éloignées. Un des traits caractéristiques avait reçu le nom de split representation: un dessin Caduveo figurera ainsi un visage tatoué composé de deux profils accolés. Le tracé sur plan ne respecte pas les lois du trompe-l'œil qu'appellent les deux dimensions, mais choisit de reproduire sur ce support plan, sans déformation, le décor tel qu'il aurait pu être dessiné sur un visage; de là cet effet de profils accolés. Cette conservation du décor ne tient, c'est là l'interprétation de Levi-Strauss, qu'à prendre la mesure du fait qu'il «est le visage», qu'il «le crée», «lui confère son être social», qu'il n'y a nul visage «derrière»le décor. Le paradigme de ce rapport, Lévi-Strauss le trouve dans les masques à volets. Ces masques désignent magistralement qu'il n'est d'autre mode du démasquer que celui qu'ils permettent et rendent manifeste, à savoir ouvrir le masque, le dédoubler à l'envers. Démasquer n'a rien à faire avec dévoiler; c'est faire étalage du masque.

André Gide à tenir ce masque ouvert s'emploie en permanence. Il y inscrit avec les événements d'une vie qui est de toujours biographiée, ses créations littéraires dont il affirme - mais bien sûr on ne le croit pas - qu'elles étaient déjà de bonne heure, toutes embrassées. Que le masque dans l'inattendu d'un éclair aille à se refermer, que cesse, un bref temps, de s'écrire cette exclusion de la relation au semblable, ce sera alors: angoisse lorsque quelque chose rendra manifeste que ce semblable peut comme tel s'évanouir ou être désiré ou encore lorsque le voile tombe où ce semblable apparaitrait cauchemar ${ }^{19}$.

15. «Si le grain ne meurt» dans Poésies, Journal, souvenirs, NRF, 1952, p. 310.

16. Journal des Faux monnayeurs, Gallimard, p. 31.

17. Cité par A. Anglès: André Gide et le premier groupe de la NRF, Gallimard, p. 38.

18. Cl. Lévi-Strauss : Anthropologie structurale, p. 369-399.

19. J. Delay : la Jeunesse d'André Gide, p. 138-142 (cauchemar); p. 171-176 et 326 (schaudern). 
L'existence comme telle de cet objet qu'est le Moi fait pour Gide difficulté. Cette existence pour se fonder d'un certain rapport au désir de l'Autre ${ }^{20}$ ne ferait que signaler pour l'enfant Gide ce pur vide mortifère mal recouvert par cet idéal angélique dont Madame Gide se fait la servante attentive et obstinée. Que Madeleine ici n'ait finalement fait que pur et simple relais montre que de ce «mystique orient" de sa vie que fut son amour pour elle, cette vie elle-même ne put trouver différente orientation. Madeleine croira trouver ce que Freud nomme plaisir, c'est-à-dire sa tranquillité en se contentant d'incarner pour Gide la figure d'une Eurydice interdite de regard. Pourtant cet accord silencieux entre eux, une confession à demi-mot où il lui avouera ne pas partir seul en Angleterre, suffira à le déchirer avec ses lettres qu'en guise de réplique, une à une, elle jette au feu de sa féminité, mettant pour lui en question l'édification de ce masque ouvert sur lequel elles s'écrivaient. Acte «d'une vraie femme ${ }^{21}$ n'hésite pas à dire Lacan, en ce qu'il interroge directement sa castration, qu'il la provoque même à cesser de ne pas s'écrire. Acte qui souligne d'un refus la fonction même de la lettre en tant qu'elle «vient prendre la place mème d'où le désir s'est retiré». Je n'écris, précise Gide, que pour être relu; c'est là ce à quoi Madeleine, dans l'intempestif de son acte, répond d'un simple «non».

En quoi ce rapport de Gide à la lettre est-il marqué du nécessaire? Si la question est en ces termes correctement formulée, on peut attendre de la psychanalyse qu'elle ne reste pas sur ce point silencieuse? Y répondre par une boutade en avançant par exemple que nul sujet hors champ de la psychose ne saurait se soutenir comme une lune, au sens où Lacan en parle dans son Séminaire sur le Moi, pour n'être pas sans pertinence, reste insuffisant. Notons toutefois que c'est juste après ce repérage du statut des lunes dans un univers ordonné par la science que Lacan introduit le schéma $L$ en tant qu'il écrit ce que Freud fraie à nouveau, avec l'Au-delà du principe du plaisir, de l'expérience psychanalytique. Le schéma $L$ écrit le fondamental dualisme freudien, l'irréductible assimilation, la non-équivalence des deux systèmes du Moi et de l'inconscient. On ne montrera pas ici en quoi il est translittération, en un autre alphabet, du schéma du chapitre VII de la Traumdeutung, en quoi il inscrit une «structure comparable ${ }^{22}$. C'est plutôt en tant qu'écrit dont peut dépendre une lecture que le schéma $L$ nous importe présentement.

Si en effet, l'interposition de la relation imaginaire est nécessaire à maintenir la distinction du grand Autre et du petit autre à partir de laquelle le parlêtre n'est pas une lune, c'est cette distinction même que Gide s'évertue à soutenir en donnant un semblant de consistance - c'est la fonction du masque redoublé à cette ligne $a a^{\prime}$, à cette relation au semblable dont il est exclu. Non sans qu'il en résulte pour lui un mode d'adresse très particulier puisque le lieu d'où pour-

20. Séminaire de Lacan du 26.3.58 (Les formations de l'inconscient) où ce rapport s'écrit ainsi : $\mathrm{d} \rightarrow \$ \Delta \diamond \mathrm{i}(\mathrm{a}) \leftarrow \mathrm{m}$.

21. Écrits, p. 761 . Le terme ici repris se trouve accentué quelques lignes plus bas de son opposition à celui de «femelle» avec lequel Lacan épingle le gémissement de Gide au su de ce que Madeleine avait fait de ses lettres.

22. Le Moi dans la théorie de Freud et dans la technique psychanalytique, p. 129. 
rait lui faire retour son propre message est reculé à l'infini, incarné par un lecteur toujours à venir auquel il livre en vrac ces lettres qui n'ont pas de sens, lettres-fétiches, lettres en souffrance d'un transfert qui n'en finit pas. Le masque ouvert supplée au défaut de "représenter ${ }^{23}$ au sens gidien. Il ne trouve sa fonction que du point exact où ce défaut interdit la translittération. Nous retrouvons ici ce sur quoi avait débouché l'analyse de la phobie qui s'avère ainsi exemplaire : ce qui ne cesse pas de s'écrire, le nécessaire, tient à la défaillance de la translittération, ou encore: là même où ça ne satisfait pas à la structure, il n'y a de nécessité que du fait que ça doive y satisfaire quand même. Par là, le nécessaire «nécessite la rencontre de l'impossible qui ne peut s'aborder que par des lettres ${ }^{24}$.

Une clinique ne sera psychanalytique que de l'écrit.

James G. Février rapporte que recevant une missive qui comportait pour la première fois des signes-voyelles, Abd Alläh Ibn Tähir, gouverneur du Khorasan en 844 , vexé de ce que cette missive mettait en doute ses capacités de lecteur, considérant comme une impolitesse à son égard cette introduction des signes-voyelles, se serait écrié : «Quel chef-d'œuvre ce serait sans toutes ces graines de coriandre qu'on y a saupoudrées $\gg^{25}$. Humour d'un homme qui savait n'avoir pas le choix.

Hors cet appui du texte ${ }^{26}$, la clinique psychanalytique ne peut que virer au pire, ce pire que Gide n'ignorait pas lorsque, évoquant dans son Corydon ce que les médecins avaient pu barbouiller sur l'uranisme, il ne manque pas d'y relever ce qu'il nomme fort justement «une intolérable odeur de clinique ${ }^{27}$.

23. C'est-à-dire composer. Gide sait parfaitement que le roman n'est pas peinture de la réalité mais fiction de la vérité.

24. Les non-dupes errent, séminaire du 8.1.74.

25. J.G. Février: Histoire de l'écriture, Payot, p. 270.

26. «Proposition du 9 octobre 1967 », l'opposition du savoir textuel et du référentiel.

27. Corydon, Gallimard, p. 30. 